\title{
High-Temperature Analyticity in Classical Lattice Systems
}

\author{
Robert B. Israel \\ University of British Columbia, Mathematics Department, Vancouver, 8, Canada
}

\begin{abstract}
We prove analyticity of the correlation functions for classical lattice systems, including "continuous-spin" systems, at high temperatures and in strong external fields. For systems whose configuration spaces are homogeneous spaces for compact groups (e.g. Ising, plane rotator and classical Heisenberg models), improved estimates on the region of analyticity are obtained by generalizing an integral equation of Gruber and Merlini. Exponential cluster properties are also obtained for such systems with a finiterange interaction.
\end{abstract}

\section{Introduction}

Using an equation of the "Kirkwood-Salsburg" type, Gallavotti and Miracle [2] have shown that the correlation functions and thermodynamic pressure of a classical lattice gas are analytic in the interaction parameters at high temperature and low activity. A similar equation can be used to deal with models where the "spin" at each site can take on any finite number of values, as opposed to the two values 0 and 1 for the lattice gas. However, this method does not extend to "continuous-spin" models.

In this paper, we present two models of proving analyticity for more general classical lattice systems. The first (Section II) works for very general systems, and is based on a theorem of Dobrushin [1] on uniqueness of measures with given conditional probabilities. The second method (Section III) requires some additional structure on the configuration space at each site: it must admit a transitive action by a compact group of measure-preserving homeomorphisms. We can then define an integral equation, generalizing one considered by Gruber and Merlini [3] for the spin $-\frac{1}{2}$ model. This involves a "Fourier series" expansion of the correlation functions; for a classical Heisenberg model, it is an expansion in spherical harmonics. In Section IV we obtain exponential cluster properties for finite-range interactions from this equation. 


\section{Analyticity for Random Fields}

Our "lattice" will be a countable set $S$ of "sites". At each site $j$ there is a "configuration space" $\Omega_{j}$, which we assume, for the sake of simplicity, to be a compact Hausdorff space. For each subset $\Lambda$ of $S$, the Cartesian product $\Omega_{\Lambda}=\prod_{j \in \Lambda} \Omega_{j}$ (with the product topology) is the configuration space for $\Lambda$. We abbreviate $\Omega_{S}$ as $\Omega$. All "measures" will be complex Borel measures. $\mathscr{M}\left(\Omega_{\Lambda}\right)$ and $C\left(\Omega_{\Lambda}\right)$ respectively will denote the spaces of measures and complex-valued continuous functions on $\Omega_{\Lambda}$, with norms denoted by $\|\cdot\|$. The number of sites in $X \subset S$ is denoted by $|X|$.

A probability measure $\varrho$ on $\Omega$ is said to have the conditional probabilities $\mu_{j}(d s \mid x)$ for $j \in S, x \in \Omega_{\{j\}^{c}}$ if for all $f \in C(\Omega)$ and $j \in S$,

$$
\varrho(f)=\int_{\Omega_{\{j\}^{\mathrm{c}}}} \varrho(d x) \int_{\Omega_{j}} \mu_{j}(d s \mid x) f(s \times x) .
$$

Some measurability condition must be imposed on $\mu_{j}(d s \mid x)$ here; for convenience, we will assume it is continuous as a function from $\Omega_{\{j\}^{c}}$ to $\mathscr{M}\left(\Omega_{j}\right)$ with the weak*topology. In statistical mechanics the conditional probabilities are usually given, and we wish to study probability measures ("Gibbs states") with those conditional probabilities. Assuming such a measure exist (as it will in the applications to statistical mechanics; in general some consistency conditions are required), we ask first whether it is unique. One sufficient condition for this is given by Dobrushin [1]. Next, if the conditional probabilities are varied analytically, we ask whether the Gibbs state varies analytically in an appropriate sense. This leads us to study complex "conditional measures".

Note [10] that we can not expect the expectation $\varrho(f)$ of every $f \in C(\Omega)$ to vary analytically: by a well-known theorem of Dunford ([7], Theorem 76) this would imply that $\varrho$ varies continuously in the norm topology. On the other hand, in typical cases the distance between Gibbs states for different values of a parameter is always 2 . What we actually expect is that $\varrho(f)$ should be analytic for $f$ in a suitable dense subspace of $C(\Omega)$, e.g. the "local" functions: those that depend only on the configuration at a finite number of sites.

A system of complex "conditional measures" $\mu_{j}(d s \mid x)$ will be assumed to have the following properties:

(i) For each $j \in S, x \mapsto \mu_{j}(d s \mid x)$ is a continuous function from $\Omega_{\{j\}^{c}}$ to $\mathscr{M}\left(\Omega_{j}\right)$ with the weak* topology.

(ii) For each $j \in S$ and $x \in \Omega_{\{j\}}, \mu_{j}(1 \mid x)=1$.

An operator $\tau_{j}: C(\Omega) \rightarrow C\left(\Omega_{\{j\}}\right)$ is defined by

$$
\tau_{j} f(x)=\int_{\Omega_{j}} \mu_{j}(d s \mid x) f(s \times x) \text { for } x \in \Omega_{\{j\}^{c}} .
$$

Later we will write $\mu_{j}^{(z)}(d s \mid x)$, and correspondingly $\tau_{j}^{(z)}$, depending on a parameter $z$. If the $\mu_{j}(d s \mid x)$ are probability measures, $\tau_{j}$ is a contraction on $C(\Omega)$, but in the general case it is more difficult to control. We now introduce a space of functions with a norm in which $\tau_{j}$ will be a contraction under certain conditions.

Let $r>0$. We define $\mathscr{E}$ ar as the space of functions $f \in C(\Omega)$ which can be written as $f=\sum_{X} f_{X}$ (the sum being over finite subsets of $S$ ), with $f_{X} \in C\left(\Omega_{X}\right)$ and

$$
\sum_{X} e^{r|X|}\left\|f_{X}\right\|<\infty .
$$


A norm on $\mathscr{E}$ is given by

$$
\|\| f \|_{r} \equiv \inf \left\{\sum_{X} e^{r|X|}\left\|f_{X}\right\|: f=\sum_{X} f_{X}, f_{X} \in C\left(\Omega_{X}\right)\right\} .
$$

Note that $\|f\| \leqq\|f\|_{r}$, and that $\mathscr{E}^{r}$ is a Banach algebra with pointwise multiplication and the norm $\|\cdot \cdot\|_{r}$.

Lemma II.1. Suppose there are continuous functions $y \mapsto \mu_{j}^{Y}(d s \mid y)$ from $\Omega_{Y}$ to $\mathscr{M}\left(\Omega_{j}\right)$ with the weak* topology, such that

$$
\mu_{j}(d s \mid x)=\sum_{Y \subset\{j\}^{c}} \mu_{j}^{Y}(d s \mid x) \quad \text { for } \quad x \in \Omega_{\{j\}^{c}} \quad \text { and } \quad \sum_{Y} e^{r(|Y|-1)} \sup _{y \in \Omega_{Y}}\left\|\mu_{j}^{Y}(d s \mid y)\right\| \leqq 1 .
$$

Then $\tau_{j}$ is a contraction on $\mathscr{E}$.

Proof. If $f=\sum_{X} f_{X}$ as above,

$$
\begin{aligned}
\left\|\tau_{j} f\right\|_{r} & \leqq \sum_{X \ngtr j}\left\|f_{X}\right\|_{r}+\sum_{X \ni j} \sum_{Y} \mid\left\|\int \mu_{j}^{Y}(d s \mid \cdot) f_{X}(s \times \cdot)\right\|_{r} \\
& \leqq \sum_{X \ngtr j} e^{r|X|}\left\|f_{X}\right\|+\sum_{X_{\ni j}} \sum_{Y} e^{r(|X|+|Y|-1)}\left\|\int \mu_{j}^{Y}(d s \mid \cdot) f_{X}(s \times \cdot)\right\| \\
& \leqq \sum_{X_{\nexists j}} e^{r|X|}\left\|f_{X}\right\|+\left(\sum_{X \ni j} e^{r|X|}\left\|f_{X}\right\|\right)\left(\sum_{Y} e^{r(|Y|-1)} \sup _{y \in \Omega_{Y}}\left\|\mu_{j}^{Y}(d s \mid y)\right\|\right) .
\end{aligned}
$$

For distinct sites $i, j \in S$, let

$$
\varrho_{j i}=\frac{1}{2} \sup \left\{\left\|\mu_{j}(d s \mid x)-\mu_{j}\left(d s \mid x^{\prime}\right)\right\|: x, x^{\prime} \in \Omega_{\{j\}^{c}}, x_{k}=x_{k}^{\prime} \text { for } k \neq i\right\} .
$$

In [1], Dobrushin showed that if the $\mu_{j}(d s \mid x)$ are probability measures and there is $\alpha<1$ such that $\sum_{i \in\{j\}^{c}} \varrho_{j i} \leqq \alpha$ for all $j \in S$, then there is at most one measure with conditional probabilities $\mu_{j}(d s \mid x)$. This condition is related to the hypotheses of Lemma II.1 as follows:

Lemma II.2. Suppose the hypotheses of Lemma II.1 hold with (1) replaced by

$$
\sum_{Y} e^{r(|Y|-1)} \sup _{y \in \Omega_{Y}}\left\|\mu_{j}^{Y}(d s \mid y)\right\| \leqq \alpha<1 .
$$

Then $\sum_{i \in\{j\}^{c}} \varrho_{j i} \leqq \alpha$.

Proof. Let $a_{Y}=\sup _{y \in \Omega_{Y}}\left\|\mu_{j}^{Y}(d s \mid y)\right\|$. Then $\varrho_{j i} \leqq \sum_{Y \ni i} a_{Y}$, so that $\sum_{i \in\{j\}^{c}} \varrho_{j i} \leqq \sum_{Y}|Y| a_{Y}$. Let $f(t)=\sum_{Y} e^{t|Y|} a_{Y}-\alpha e^{t}$. By assumption $f(r) \leqq 0$, while $f(0) \geqq 0$ since $\mu_{j}(1 \mid x)=1$. Therefore for some $t>0$ we have

$$
0 \geqq f^{\prime}(t)=\sum_{Y}|Y| a_{Y} e^{t|Y|}-\alpha e^{t} \geqq e^{t}\left(\sum_{Y}|Y| a_{Y}-\alpha\right) .
$$

In his proof [6] of Dobrushin's result, Lanford uses the space $\mathscr{F}$ of functions $f \in C(\Omega)$ with $\sum_{i \in S} \delta_{i}(f)<\infty$, where

$$
\delta_{i}(f)=\sup \left\{\left|f(x)-f\left(x^{\prime}\right)\right|: x, x^{\prime} \in \Omega, x_{k}=x_{k}^{\prime} \text { for } k \neq i\right\} .
$$

It is easily seen that our space $\mathscr{E}$ is contained in $\mathscr{F}$. 
Theorem II.3. Let $\mu_{j}^{(z)}(d s \mid)$ depend analytically on the parameter $z$ in some open subset $W$ of $\mathbb{C}^{n}$ (so that for fixed $x$ and $j$, the $\mathscr{M}\left(\Omega_{j}\right)$-valued function $z \rightarrow \mu_{j}^{(z)}(d s \mid x)$ is analytic). Assume that:

(i) For each $z \in W$ there are $\alpha<1$ and $\mu_{j}^{Y}(d s \mid y)$ as in Lemma II.1 for which (2) is satisfied.

(ii) $W \cap \mathbb{R}^{n}$ is a set of uniqueness for $W$.

(iii) For $z \in W \cap \mathbb{R}^{n}$ the $\mu_{j}^{(z)}(d s \mid x)$ are probability measures, and are the conditional probabilities for some probability measure $\varrho^{(z)}$ (which is unique by Dobrushin's result).

Then if $f \in \mathscr{E}$ there is an analytic function $g(z)$ on $W$ with $|g| \leqq\|f f\|_{r}$ and $g(z)=$ $\varrho^{(z)}(f)$ for $z \in W \cap \mathbb{R}^{n}$.

Proof. As shown in ([6], p. 110), for each $z \in W \cap \mathbb{R}^{n}$ and $f \in \mathscr{F}$ there is a sequence $\left(j_{n}\right)$ in $S$ for which

$$
\sum_{i \in S} \delta_{i}\left(\tau_{j_{n}}^{(z)} \ldots \tau_{j_{1}}^{(z)} f\right) \rightarrow 0
$$

Since

$$
\sum_{i \in S} \delta_{i}\left(\tau_{j}^{(z)} f\right) \leqq \sum_{i \in S} \delta_{i}(f) \quad \text { for } \quad f \in \mathscr{F} \quad \text { and } \quad z \in W \cap \mathbb{R}^{n}
$$

it is easily seen that given $f \in \mathscr{F}$ and a countable dense subset $C$ of $W \cap \mathbb{R}^{n}$, there is a sequence $\left(j_{n}\right)$ in $S$ for which (3) holds for all $z \in C$. Now fix $x \in \Omega, f \in \mathscr{E}^{r}$, and consider the sequence of analytic functions of $z \in W$ given by

$$
\tau_{j_{n}}^{(z)} \ldots \tau_{j_{1}}^{(z)} f(x)
$$

By Lemma II.1, these are uniformly bounded by $\|f\|_{r}$. For $z \in C$, they converge to $\varrho^{(z)}(f)$ as in Lanford's proof. By Vitali's Theorem, they converge uniformly on compact subsets of $W$ to an analytic function $g(z)$, which agrees with $\varrho^{(z)}(f)$ on $C$. Note that $g(z)$ is independent of the choice of $x$, since this is true on the set of uniqueness $C$. Therefore, for $z \in W \cap \mathbb{R}^{n}$ we have

$$
\varrho^{(z)}(f)=\lim _{n \rightarrow \infty} \varrho^{(z)}\left(\tau_{j_{n}}^{(z)} \ldots \tau_{j_{1}}^{(z)} f\right)=g(z) .
$$

The conditional measures in statistical mechanics arise from "interactions". Let $\tilde{\mathscr{B}}_{r}$ be the space of (complex) interactions $\Phi$ defined by the following conditions:

(i) For each nonempty finite subset $X$ of $S, \Phi(X)$ is a complex-valued function in $C\left(\Omega_{X}\right)$.

(ii) The norm in $\tilde{\mathscr{B}}_{r}$ is $\|\Phi\|_{r}=\sup _{j \in S} \sum_{X \ni j_{\tilde{\sim}}} e^{r(|X|-1)}\|\Phi(X)\|<\infty$. The space of real interactions in $\tilde{\mathscr{B}}_{r}$ is denoted $\mathscr{B}_{r}$. For $\Phi \in \mathscr{\mathscr { B }}_{r}$ we define "conditional Hamiltonians"

$$
H_{j}^{\Phi}(s \mid x)=\sum_{X \ni j} \Phi(X)(s \times x) \quad s \in \Omega_{j}, \quad x \in \Omega_{\{j\}^{\mathrm{c}}}
$$

and the corresponding conditional measures

$$
\mu_{j}^{\Phi}(d s \mid x)=\frac{e^{-H_{j}^{\Phi}(s \mid x)} v_{j}(d s)}{\int_{\Omega_{j}} e^{-H_{j}^{\Phi}\left(s^{\prime} \mid x\right)} v_{j}\left(d s^{\prime}\right)}
$$


where $v_{j}$ is a fixed "a priori" probability measure on $\Omega_{j}$, and the denominator in (5) is assumed to be nonzero.

It will be convenient to modify the algebra $\mathscr{E}^{\mathscr{r}}$ and its norm by giving special treatment to a fixed site $j$, leaving it out of the exponential factor in the norm. Thus we define

$$
\|\| f \|_{r, j}=\inf \left\{\sum_{X} e^{r|X \backslash j|}\left\|f_{X}\right\|: f=\sum_{X} f_{X}, f_{X} \in C\left(\Omega_{X}\right)\right\}
$$

and $\mathscr{E}^{r, j}=\left\{f \in C(\Omega):\|f\|_{r, j}<\infty\right\}$. This is clearly still a Banach algebra.

The following theorem shows that at "high temperature" Theorem II.3 is applicable.

Theorem II.4. Let $\Phi=\Psi+\hat{\Phi} \in \tilde{\mathscr{B}}_{r}$, where $\Psi$ is real with $\Psi(X)=0$ for $|X|>1$, while $\|\hat{\Phi}\|_{r}<\ln \left(2 /\left(1+e^{-r}\right)\right)$. Then there are $\alpha<1$ and $\mu_{j}^{Y}(d s \mid y)$ for which condition (2) is satisfied. If $W$ is the set of $\Phi$ as above, then the Gibbs state $\varrho^{\Phi}$ is unique for $\Phi \in W \cap \mathscr{B}_{r}$, and for $f \in \mathscr{E}$ the function $\Phi \rightarrow \varrho^{\Phi}(f)$ extends to a function $g_{f}(\Phi)$ on $W$ which is analytic on $W \cap \mathscr{N}$ for each finite-dimensional complex linear subspace $\mathscr{N}$ of $\tilde{\mathscr{B}}_{r}$, with $\left|g_{f}(\Phi)\right| \leqq\|f\|_{\text {r. }}$.

Proof. We can ignore $\Psi$, since its only effect is to change $v_{j}(d s)$ to a new probability measure

$$
\frac{e^{-\Psi(\{j\})(s)} v_{j}(d s)}{\int_{\Omega_{j}} e^{-\Psi(\{j\})\left(s^{\prime}\right)} v_{j}\left(d s^{\prime}\right)} .
$$

Therefore we will take $\Psi=0$. Let

$$
h_{j}(s \mid x)=\frac{e^{-H_{j}^{\Phi}(s \mid x)}}{\int_{\Omega_{j}} e^{-H_{j}^{\Phi}\left(s^{\prime} \mid x\right)} v_{j}\left(d s^{\prime}\right)} .
$$

We need to estimate $\left\|h_{j}\right\|_{r, j}$. It is clear that

$$
\left\|e^{-H_{j}^{\Phi}}\right\|_{r, j} \leqq e^{\|\| H_{j}^{\Phi}\|\|_{r, j}} \leqq e^{\|\Phi\|_{r}} .
$$

Moreover

$$
\begin{aligned}
\|\| 1-\int_{\Omega_{j}} e^{-H_{j}^{\Phi}\left(s^{\prime} \mid \cdot\right)} v_{j}\left(d s^{\prime}\right) \mid \|_{r, j} & =\left\|\int_{\Omega_{j}}\left(1-e^{-H_{j}^{\Phi}\left(s^{\prime} \mid \cdot\right)}\right) v_{j}\left(d s^{\prime}\right)\right\| \|_{r, j} \\
& \leqq\left\|1-e^{-H_{j}^{\Phi}}\right\|_{r, j} \leqq e^{\|\Phi\|_{r}}-1 .
\end{aligned}
$$

If the latter is less than $1, \int_{\Omega_{j}} e^{-H_{j}^{\Phi}\left(s^{\prime} \mid \cdot\right)} v_{j}\left(d s^{\prime}\right)$ is invertible and

$$
\left\|\left(\| \int_{\Omega_{j}} e^{-H_{J}^{\Phi}\left(s^{\prime} \mid \cdot\right)} v_{j}\left(d s^{\prime}\right)\right)^{-1}\right\| \|_{r, j} \leqq \frac{1}{2-e^{\|\Phi\|_{r}}} .
$$

Thus we obtain $\left\|h_{j}\right\|_{r, j} \leqq \frac{e^{\|\Phi\|_{r}}}{2-e^{\|\Phi\|_{r}}}<e^{r}$

if

$$
\|\Phi\|_{r}<\ln \frac{2}{1+e^{-r}} .
$$

The rest follows directly from Theorem II.3. 
Theorem II.4 was independent of the one-body ("chemical potential" or "external magnetic field") terms. Next we will show how analyticity can be obtained by adding to a given interaction a sufficiently strong one-body term favoring one configuration. Although it is possible to obtain somewhat greater generality, it is convenient to formulate this result in a "translation-invariant" context (to ensure that certain estimates are uniform on $S$ ). Thus we assume that $S$ is a group, and that all the $\Omega_{j}$ are copies of each other, with the same "a priori" measure $v_{j}$. Thus we have a group of "translations" $\alpha_{j}$ on $C(\Omega)$, with $\alpha_{j}\left(C\left(\Omega_{\Lambda}\right)\right)=$ $C\left(\Omega_{j \cdot A}\right)$ and $v_{j \cdot i} \circ \alpha_{j}=v_{i \cdot}$. The interaction $\Phi$ is said to be translation-invariant if

$\Phi(j \cdot X)=\alpha_{j}(\Phi(X))$ for all $j \in S$ and $X \subset S$ finite.

Spaces of translation-invariant interactions will be denoted by $\mathscr{B}_{r}^{I}$ and $\tilde{\mathscr{B}}_{r}^{I}$.

Theorem II.5. Let $\Phi \in \tilde{\mathscr{B}}_{r}^{I}$, and $\Psi \in \mathscr{B}_{r}^{I}$ with $\Psi(X)=0$ for $|X|>1$, such that $\Psi(\{j\})$ attains its minimum at exactly one point $s_{0}$, which is in the support of $v_{j}$. Then for $t>0$ sufficiently large, there is a neighborhood $W$ of $\Phi+t \Psi$ in $\tilde{\mathscr{B}}_{r}$ in which the conclusions of Theorem II.4 are valid.

Proof. As before, the effect of $\Psi$ is to change $v_{j}$. We let

$$
v_{j}^{t}(d s)=\frac{e^{-t \Psi((j j)(s)} v_{j}(d s)}{\int_{\Omega_{J}} e^{-t \Psi\left((j j)\left(s^{\prime}\right)\right.} v_{j}\left(d s^{\prime}\right)}
$$

and

$$
h_{j}^{t}(s \times x)=\frac{e^{-H_{j}^{\Phi}(s \times x)}}{\int_{\Omega_{j}} e^{-H_{J}^{\Phi}\left(s^{\prime} \times x\right)} v_{j}^{t}\left(d s^{\prime}\right)} .
$$

Given $\delta>0$, there is a neighborhood $U$ of $s_{0}$ such that

$$
\sum_{X \ni j} e^{r(|X|-1)}\left|\Phi(X)(s \times x)-\Phi(X)\left(s_{0} \times x\right)\right|<\delta \quad \text { for } \quad s \in U, \quad x \in \Omega_{\{j\}^{c}} .
$$

For $t$ sufficiently large, $v_{j}^{t}(U)>1-\delta$. We then have

$$
\begin{aligned}
& \|\| 1-e^{H_{j}^{\Phi}\left(s_{0} \mid \cdot\right)} \int_{\Omega_{j}} e^{-H_{j}^{\Phi}\left(s^{\prime} \mid \cdot\right)} v_{j}^{t}\left(d s^{\prime}\right)\|\|_{r, j} \\
& \leqq \int_{U}\left|\left\|1-e^{H_{j}^{\Phi}\left(s_{0} \mid \cdot\right)-H_{j}^{\Phi}\left(s^{\prime} \mid \cdot\right)} \mid\right\|_{r, j} v_{j}^{t}\left(d s^{\prime}\right)+\delta\left(e^{2\|\Phi\|_{r}}-1\right)\right. \\
& \leqq e^{\delta}-1+\delta\left(e^{2\|\Phi\|_{r}}-1\right) \equiv \varepsilon .
\end{aligned}
$$

Thus we can write $h_{j}^{t}=\sum_{Y \ni j} h_{Y}$ with $h_{Y} \in C\left(\Omega_{Y}\right)$ and

$$
\sum_{Y} e^{r|Y \backslash j|}\left\|h_{Y}\right\| \leqq(1-\varepsilon)^{-1} e^{2\|\Phi\|_{r}}
$$

while

$$
\sum_{Y} e^{r|Y \backslash j|}\left\|h_{Y}(s \times \cdot)\right\| \leqq(1-\varepsilon)^{-1}\left\|e^{H_{j}^{\Phi}\left(s_{0} \mid \cdot\right)-H_{j}^{\Phi}(s \mid \cdot)}\right\|_{r, j}<(1-\varepsilon)^{-1} e^{\delta} \quad \text { for } \quad s \in U .
$$


Taking $\mu_{j}^{Y}(d s \mid y)=h_{Y \cup\{j\}}(s \times y) v_{j}^{t}(d s)$, we have

$$
\sum_{Y} e^{r|Y|} \sup _{y \in \Omega_{Y}}\left\|\mu_{j}^{Y}(d s \mid y)\right\| \leqq(1-\varepsilon)^{-1}\left(e^{\delta}+\delta e^{2\|\Phi\|_{r}}\right)<e^{r}
$$

if $\delta$ is chosen sufficiently small.

If $S=\mathbb{Z}^{v}$ there is a continuous convex function $P$ on $\mathscr{B}_{r}^{I}$ traditionally called the "pressure", and the translation-invariant Gibbs states are obtained from functionals tangent to its graph (see [6]). Whenever the invariant Gibbs state for $\Phi$ is unique,

$$
\frac{d}{d t} P\left(\Phi+t \Phi^{\prime}\right)_{\mid t=0}=-\varrho^{\Phi}\left(A_{\Phi^{\prime}}\right)
$$

where

$$
A_{\Phi^{\prime}}=\sum_{X \ni 0} \frac{\Phi^{\prime}(X)}{|X|} .
$$

Therefore we have.

Corollary II.6. If $S=\mathbb{Z}^{v}$ and $W \subset \tilde{\mathscr{B}}_{r}^{I}$ is an open starshaped set in which the conclusions of Theorem II.4 are valid, then the pressure $P$ extends from $W \cap \mathscr{B}_{r}^{I}$ to a function on $W$ which is analytic on $W \cap \mathscr{N}$ for each finite-dimensional complex linear subspace $\mathscr{N}$ of $\tilde{\mathscr{B}}_{r}^{I}$.

Proof. Consider $g_{A_{\Phi}}(\Phi)$ as a holomorphic differential 1-form on $W$; on $W \cap \mathscr{B}_{r}^{I}$ it is the derivative of the pressure, so by analytic continuation it is a closed form, and we can integrate it.

\section{An Integral Equation}

With additional structure on the configuration space, we can replace $\mathscr{E}$ by a Banach algebra of absolutely converging Fourier series, and obtain a linear equation in the dual of this algebra which the Gibbs state must satisfy. This will give us a "high temperature" result for a somewhat different space of interactions: the exponential factor in the norm is no longer needed, but the uniform norm of $\Phi(X)$ is replaced by the $\ell^{1}$ norm of its Fourier series. In most cases the bounds on critical temperatures obtained by this method are much better than those given by Theorem II.4.

For simplicity, we first consider the case where the configuration spaces $\Omega_{i}$, and thus also the product $\Omega$, are compact abelian groups, with normalized Haar measure denoted $d s$. A character of $\Omega$ is either 1 or of the form $\gamma_{X}=\gamma_{i_{1}} \otimes \ldots \otimes \gamma_{i_{n}}$ where $X=\left\{i_{1}, \ldots, i_{n}\right\}$ is a finite subset of $S$ and $\gamma_{i_{j}} \neq 1$ is a character of $\Omega_{i_{j}}$. The dual groups of $\Omega$ and $\Omega_{i}$ will be denoted by $\Gamma$ and $\Gamma_{i}$ respectively. Let $A(\Omega)$ be the space of complex functions $f$ on $\Omega$ with absolutely convergent Fourier series, i.e. $f=\sum_{\gamma \in \Gamma} a_{\gamma} \gamma$ with $\|f\| \equiv \sum_{\gamma \in \Gamma}\left|a_{\gamma}\right|<\infty$. The dual $A(\Omega)^{*}$ of $A(\Omega)$ is naturally identified with the space $\ell^{\infty}(\Gamma)$ of bounded sequences indexed by $\Gamma$, with the uniform norm. It is well known that $A(\Omega)$ is a Banach algebra under pointwise operations. 
Consider a system of conditional measures $\mu_{j}(d s \mid x)$ as in Section II, and suppose each $\mu_{j}\left(\gamma_{j} \mid \cdot\right) \in A(\Omega)$ with sup $\left\|\mu_{j}\left(\gamma_{j} \mid \cdot\right)\right\| \|<$. Then $\tau_{j}$ is a bounded operator on $A(\Omega)$ with norm $\sup _{\gamma_{j} \in \Gamma_{j}}\left\|\mu_{j}\left(\gamma_{j}|\cdot|^{\gamma_{j} \in \Gamma_{j}}\right)\right\|$. Let $S$ be ordered so that each finite subset $X$ is given a first element. Then we can define a linear operator $K$ on $A(\Omega)$ by

$$
\begin{aligned}
K \gamma_{X} & =\tau_{j} \gamma_{X} \quad \text { where } j \text { is the first element of } X \\
K 1 & =0 .
\end{aligned}
$$

It is clear that $K$ has norm at most $\sup \sup \left\|\mu_{j}\left(\gamma_{j} \mid \cdot\right)\right\|$. Our "integral equation" is $j \in S \quad \gamma_{j} \neq 1$

$$
K^{*} \varphi+\delta=\varphi
$$

where $\delta(1)=1$ and $\delta\left(\gamma_{X}\right)=0$ for all other characters. If the $\mu_{j}(d s \mid x)$ are probability measures, any Gibbs state must satisfy (7). This is the equation studied by Gruber and Merlini [3] for the spin $-\frac{1}{2}$ case $\left(\Omega_{j}=\mathbb{Z}_{2}\right)$. If $1-K^{*}$ is invertible, the unique solution of (7) is given by $\varphi=\left(1-K^{*}\right)^{-1} \delta$. If in addition the conditional measures depend analytically on a parameter $z \in W \subset \mathbb{C}^{n}$, then the operator $K(z)$ is also analytic in $z$, and in the open set where $1-K(z)$ is invertible, $\varphi(z)=\left(1-K(z)^{*}\right)^{-1} \delta$ is also analytic.

We define new spaces $\mathscr{B}_{A}$ and $\tilde{\mathscr{B}}_{A}$ of real and complex interactions respectively, by requiring $\Phi(X) \in A\left(\Omega_{X}\right)$ with the norm

$$
\|\Phi\| \equiv \sup _{j \in S} \sum_{X \ni j}\|\Phi(X)\|<\infty .
$$

The conditional Hamiltonians and conditional measures are as in (4) and (5), but we require $v_{j}(d s)$ to be Haar measure $d s$.

Theorem III.1. Suppose $\Phi \in \tilde{\mathscr{B}}_{A}$ with $\|\Phi\|<\ln \frac{3}{2}$. Then the operator $K(\Phi)$ of (6) has norm less than 1 . For $f \in A(\Omega)$ the function $\varphi^{\Phi}(f)$ is analytic on the intersection of the set $W$ of such $\Phi$ with any finite-dimensional complex linear subspace of $\tilde{\mathscr{B}}_{A}$ (where $\varphi^{\Phi}$ is the unique solution of the equation $K(\Phi)^{*} \varphi+\delta=\varphi$ ).

Proof. For $\gamma_{j} \neq 1$ we have

$$
\left\|\mu_{j}^{\Omega}\left(\gamma_{j} \mid \cdot\right)\right\|=\left\|\frac{\int_{\Omega_{j}}\left(1-e^{-H_{j}^{\Phi}(s \mid \cdot)}\right) \gamma_{j}(s) d s}{\int_{\Omega_{j}} e^{-H_{j}^{\Phi}(s \mid \cdot)} d s}\right\| \leqq \frac{\left\|1-e^{-H_{j}^{\Phi}}\right\|}{1-\left\|1-e^{-H_{j}^{\Phi}}\right\|}
$$

and

$$
\left\|1-e^{-H_{j}^{\Phi}}\right\| \mid \leqq e^{\left\|H_{j}^{\Phi}\right\| \|}-1 \leqq e^{\|\Phi\|}-1<\frac{1}{2} .
$$

In many cases we can obtain improved estimates on $\left\|\mu_{j}^{\Phi}\left(\gamma_{j} \mid\right)\right\| \|$. The simplest is the spin $-\frac{1}{2}$ Ising model with interaction of the form

$$
\Phi(X)=-J(X) \sigma_{X}\left(J(X) \in \mathbb{C},\|\Phi\|=\sup _{j \in S} \sum_{X \ni j}|J(X)|<\infty\right) .
$$


Letting $u_{j}=\sum_{X \ni j} J(X) \sigma_{X \backslash j}$, we obtain $\mu_{j}\left(\sigma_{j} \mid \cdot\right)=\tanh u_{j}$. Now in the Taylor series $\tanh z=\sum_{n=0}^{\infty} b_{n} z^{2 n+1}$, the sign of $b_{n}$ is $(-1)^{n}$. Thus

$$
\left\|\left|\tanh u_{j}\left\|\leqq \sum_{n=0}^{\infty}\left|b_{n}\right|\right\| u_{j}\|\|^{2 n+1}=\frac{1}{i} \tanh \left(i\left\|u_{j}\right\|\right)=\tan \left\|u_{j}\right\| \| .\right.\right.
$$

Thus the conclusions of Theorem III.1 hold for this model if

$$
\sup _{j \in S}\left\|u_{j}\right\|(=\|\Phi\|)<\frac{\pi}{3} \text {. }
$$

On the other hand, the "mean field" value is $\|\Phi\|=1$; it follows from Dobrushin's result quoted in Section II that if $\Phi$ is a real pair interaction with $\|\Phi\|<1$ (and in general if $\left.\sup _{j \in S} \sum_{X \ni j}(|X|-1) \tanh |J(X)|<1\right)$ then $\Phi$ has a unique Gibbs state.

The plane rotator model can be treated in a similar fashion. Here $\Omega_{j}$ is the unit circle with characters $\sigma_{j}^{n}=e^{i n \theta_{j}}$. We restrict our attention to the usual pair interaction, of the form

$$
\Phi(\{i, j\})\left(\theta_{i}, \theta_{j}\right)=-J(i, j) \sigma_{1} \cdot \sigma_{2}=-\frac{1}{2} J(i, j)\left(\sigma_{i} \sigma_{j}^{-1}+\sigma_{i}^{-1} \sigma_{j}\right) .
$$

For convenience we also take the $J(i, j)$ real. Now if we write

$$
\sum_{X \ni j} J(i, j) \sigma_{i}=u_{j}=r e^{i \theta}
$$

we obtain

$$
\mu_{j}\left(\sigma_{j}^{k} \mid \cdot\right)=e^{i k \theta} \frac{I_{k}(r)}{I_{0}(r)}
$$

where $I_{k}$ is the $k$-th order modified Bessel function

$$
\begin{aligned}
& I_{k}(r)=\sum_{n=0}^{\infty} \frac{(r / 2)^{2 n+k}}{n !(n+k) !}(k \geqq 0) \\
& I_{-k}(r)=I_{k}(r) .
\end{aligned}
$$

The Taylor series $I_{k}(z) / I_{0}(z)=\sum_{n=0}^{\infty} b_{n}(k) z^{2 n+k}$ (for $k>0$ ) has coefficients $b_{n}(k)$ of $\operatorname{sign}(-1)^{n}$, so that

$$
\left\|\mu_{j}\left(\sigma_{j}^{k} \mid \cdot\right)\right\| \leqq \sum_{n=0}^{\infty}\left|b_{n}(k)\right|\left\|u_{j}\right\| \|^{2 n+k}=\frac{J_{k}\left(\left\|u_{j}\right\|\right)}{J_{0}\left(\left\|u_{j}\right\|\right)}
$$

where $J_{k}(z)=i^{k} I_{k}(-i z)$ is the $k$-th order (unmodified) Bessel function. For $0 \leqq z \leqq 2$ this is a decreasing function of $|k|$. Numerically solving $J_{0}(z)=J_{1}(z)$, we find that for this model the conclusions of Theorem III.1 hold (in some neighborhood of $\Phi)$ if

$$
\sup _{j \in S} \sum_{i \in S}|J(i, j)|<1.4347 \text {. }
$$

This compares to a "mean field" value of 2 for this model. 
Another interesting example is a "continuous Ising model" with the interval $[-1,1]$ (with normalized Lebesgue measure) as configuration space at each site, and an interaction of the form

$$
\Phi(\{i, j\})\left(s_{i}, s_{j}\right)=-J(i, j) s_{i} s_{j} .
$$

We can not use the usual Fourier series on the interval, because the interaction terms do not have absolutely convergent Fourier series. Instead, we use an expansion in Walsh functions: these are essentially the characters of $\prod_{n=1}^{\infty} \mathbb{Z}_{2}$, which is mapped onto $[-1,1]$ by the measure-preserving transformation $\left(\sigma_{n}\right) \mapsto \sum_{n=1}^{\infty} 2^{-n} \sigma_{n}$. A similar analysis to that used for the spin $-\frac{1}{2}$ model can be performed, with the result that the conclusions of Theorem III.1 hold if

$$
\sup _{j \in S} \sum_{i \neq j}|J(i, j)|<\frac{\pi}{2} .
$$

This compares to a mean field value of 3 for this model.

The estimates for these models can be improved still more for interactions of "nearest-neighbor" type, as was done for the spin $-\frac{1}{2}$ model in [3].

Now we go to the non-abelian case. Here $\Omega_{j}$ is assumed to admit a transitive action by a compact group $G_{j}$ of homeomorphisms preserving the a priori measure $v_{j}$; thus it can be identified with $G_{j} / H_{j}$ where $H_{j}$ is the isotropy subgroup of an arbitrary point, and $v_{j}$ is the probability measure induced by Haar measure on $G_{j}$ (therefore we will write it as $d s$ ). Similarly, $\Omega$ is identified with $G / H$, where $G=\prod_{j \in S} G_{j}$ and $H=\prod_{j \in S} H_{j}$. To replace the dual group of the abelian case, we have the continuous irreducible unitary representations of $G$. Let $\Sigma_{j}$ be the set of equivalence classes (for unitary equivalence) of irreducible unitary representations of $G_{j}$. For each $\sigma \in \Sigma_{j}$ we suppose some member $U^{(\sigma)}$ of $\sigma$ has been chosen. The equivalence classes of irreducible unitary representations of $G$ are given by sequences $\sigma=\left(\sigma_{j}\right)_{j \in S}$ where all but finitely many $\sigma_{j}=1$ (the trivial representation), $\sigma$ denoting the equivalence class of the representation $\bigotimes U^{\left(\sigma_{j}\right)} \equiv U^{(\sigma)}([4]$, Theorem 27.43). The set of such equivalence classes is denoted ${ }^{j} \Sigma$.

The space $A(G)$ of functions on $G$ with absolutely converging Fourier series consists of functions of the form

$$
f=\sum_{\sigma} \operatorname{Tr}\left(A_{\sigma} U^{(\sigma)}\right) \quad \text { with } \quad\|f\| \equiv \sum_{\sigma}\left\|A_{\sigma}\right\|_{1}<\infty
$$

where $A_{\sigma}$ is an operator in the representation space $\mathscr{H}_{\sigma}$, and $\|A\|_{1}$ is the trace-class norm $\operatorname{Tr}|A|$. See [4], $\S 34$, or [5]. The space $A(\Omega)$ of absolutely converging Fourier series on $\Omega$ consists of functions $f$ on $\Omega$ such that $f \circ q \in A(G)$, where $q: G \rightarrow \Omega$ is the quotient map. The norm on $A(\Omega)$ is $\|f\|=\|f \circ q\|$. An equivalent formulation is as follows: for each $\sigma$, define a function $W^{(\sigma)}$ from $\Omega$ to operators on $\mathscr{H}_{\sigma}$ by

$$
W^{(\sigma)}(g H)=\int_{H} d h U^{(\sigma)}(g h)=U^{(\sigma)}(g) P^{(\sigma)}
$$


where $P^{(\sigma)}=\int_{H} d h U^{(\sigma)}(h)$ is an orthogonal projection. Then $A(\Omega)$ consists of all functions of the form

$$
f=\sum_{\sigma} \operatorname{Tr}\left(C_{\sigma} W^{(\sigma)}\right) \quad \text { with } \quad P^{(\sigma)} C_{\sigma}=C_{\sigma} \quad \text { and } \quad\|f\|\left\|\equiv \sum_{\sigma}\right\| C_{\sigma} \|_{1}<\infty .
$$

$A(G)$ and $A(\Omega)$ are commutative Banach algebras under pointwise operations, and are dense in $C(G)$ and $C(\Omega)$ respectively (see [5]).

Suppose our conditional measures $\mu_{j}(d s \mid x)$ are of the form $g_{j}(s \times x) d s$ with $g_{j} \in A(\Omega)$. Then $\tau_{j}$ is an operator on $A(\Omega)$ of norm at most $\left\|g_{j}\right\|$. A linear operator $K$ on $A(\Omega)$ is defined by

$$
\begin{aligned}
& K 1=0 \\
& K f_{X}=\tau_{j} f_{X} \quad \text { where } \quad j \quad \text { is the first element of } X
\end{aligned}
$$

and $f_{X}=\operatorname{Tr}\left(C_{\sigma} W^{(\sigma)}\right), X=\left\{i \in S: \sigma_{i} \neq 1\right\}$. This has norm at most sup $\left\|g_{j}\right\|$. Again we have an "integral equation" as in (7), and Banach spaces $\mathscr{B}_{A}$ and $\mathscr{\mathscr { B }}_{A}^{j \in S}$ of interactions with $\Phi(X) \in A\left(\Omega_{X}\right)$.

Theorem III.2. The results of Theorem III.1 hold also for the non-abelian case as formulated above.

Proof. Suppose $f_{X}=\operatorname{Tr}\left(C_{\sigma} W^{(\sigma)}\right) \in A(\Omega)$ with $X=\left\{i \in S: \sigma_{i} \neq 1\right\}$, and $j$ is the first element of $X$. Then $\int_{\Omega_{j}} f_{X}(s \times \cdot) d s=0$, so that

$$
\left\|\tau_{j} f_{X}\right\|=\left|\left\|\frac{\int_{\Omega_{j}}\left(1-e^{-H_{j}^{\Phi}(s \mid \cdot)}\right) f_{X}(s \times \cdot) d s}{\int_{\Omega_{j}} e^{-H_{j}^{\Phi}(s \mid \cdot)} d s}|| \leqq \frac{\left\|1-e^{-H_{j}^{\Phi}}\right\|}{1-\left\|1-e^{-H_{j}^{\Phi}}\right\|}\right\| f_{X} \| .\right.
$$

As in Theorem III.1, for $\|\Phi\| \mid<\ln \frac{3}{2}$ we obtain

$$
\left\|1-e^{-H_{j}^{\Phi}}\right\| \mid \leqq e^{\|\Phi\| \|}-1<\frac{1}{2} \quad \text { so that } \quad\|K(\Phi)\|<1 .
$$

An important example is the classical Heisenberg model, where $\Omega_{j}=\mathbb{S}^{2}=$ $S O(3, \mathbb{R}) / S O(2, \mathbb{R})$. Here the "Fourier series" is an expansion in spherical harmonics; if $f(x)=\sum_{\boldsymbol{i}, \boldsymbol{m}} a_{\boldsymbol{l} \boldsymbol{m}} \prod_{j} Y_{m_{j}}^{l_{j}}\left(x_{j}\right)$ then

$$
\|\| f \|=\sum_{i}\left(\prod_{j}\left(2 l_{j}+1\right)^{1 / 2}\right)\left(\sum_{m}\left|a_{l m}\right|^{2}\right)^{1 / 2} .
$$

Here we are taking $Y^{l}$ normalized so $\int_{\Phi^{2}} d s\left|Y^{l}(s)\right|^{2}=1$ (with $d s$ normalized to 1 , not $4 \pi)$; the factor $(2 l+1)^{1 / 2}$ arises because the matrix elements of $U^{(l)}$ have $L^{2}$ norm $(2 l+1)^{-1 / 2}$ by the Schur orthogonality relations. The verification that \|\|$f g\|\mid \leqq\| f\|\|\|g\|$ is recommended as an exercise in Clebsch-Gordan coefficients.

\section{Cluster Properties}

The methods of Section III can be used to yield cluster properties for the correlation functions $\varphi^{\Phi}(f)$ where $f \in A(\Omega)$. The results of this section deal with a slight 
generalization of finite-range interactions. We conjecture that results similar to those of [8] could be obtained for exponentially-decreasing interactions.

Definition. For fixed interaction $\Phi \in \tilde{\mathscr{B}}_{A}$ and subsets $X, Y$ of $S$, let $N^{\Phi}(X, Y)$ be the least $n$ for which there is a sequence $\left\{j_{0}, j_{1}, \ldots, j_{n}\right\}$ in $S$ with $j_{0} \in X, j_{n} \in Y$, such that there are sets $Z_{i} \subset S$ with $\Phi\left(Z_{i}\right) \neq 0$ and $\left\{j_{i-1}, j_{i}\right\} \subset Z_{i}$ for $i=1, \ldots, n$. (If $X \cap Y \neq \emptyset$ then $\left.N^{\Phi}(X, Y)=0\right)$.

If we are given a metric on $S$ and $\Phi$ has range at most $R$ (i.e. $\Phi(X)=0$ whenever $\operatorname{diam}(X)>R)$, then $N^{\Phi}(X, Y) \geqq R^{-1} \operatorname{dist}(X, Y)$.

In the following lemma we use for the first time the hypothesis that the selection of first elements comes for an ordering of $S$, so that if $j$ is the first element of $X$ and $j \in Y$, then $j$ is the first element of $X \cap Y$.

Lemma IV.1. If $f \in A\left(\Omega_{X}\right)$ and $g \in A\left(\Omega_{Y}\right)$ with $N^{\Phi}(X, Y)>n$ then

$$
\delta\left(K(\Phi)^{n}(f g)\right)=\sum_{k=0}^{n} \delta\left(K(\Phi)^{k} f\right) \delta\left(K(\Phi)^{n-k} g\right) .
$$

Proof. We use induction on $n$. For $n=0, \delta(f g)=\delta(f) \delta(g)$ if $X \cap Y=\emptyset$. Suppose the lemma is true for $n-1$. We can assume $f$ and $g$ are of the form $\operatorname{Tr}\left(C_{\sigma} W^{(\sigma)}\right)$ with $\sigma_{i} \neq 1$ for $i \in X$ or $Y$ respectively. If $X$ or $Y$ is empty the result is clear. Now $K(\Phi)(f g)$ is either $f \tau_{j}(g)=f K(\Phi) g$ or $g \tau_{j}(f)=g K(\Phi) f$, depending on whether the first element $j$ of $X \cup Y$ is in $Y$ or $X$ respectively. Suppose $j \in Y$. Then $\tau_{j} g \in A\left(\Omega_{Y^{\prime}}\right)$ with $N^{\Phi}\left(X, Y^{\prime}\right) \geqq N^{\Phi}(X, Y)-1$, so by the induction hypothesis

$$
\delta\left(K(\Phi)^{n}(f g)\right)=\delta\left(K(\Phi)^{n-1}(f K(\Phi) g)\right)=\sum_{k=0}^{n} \delta\left(K(\Phi)^{k} f\right) \delta\left(K(\Phi)^{n-k} g\right)
$$

where the $k=n$ term is zero since $\delta(g)=0$.

Theorem IV.2. Let $\Phi \in \mathscr{B}_{A}$ with $\|K(\Phi)\|<1, f \in A\left(\Omega_{X}\right)$ and $g \in A\left(\Omega_{Y}\right)$ with $N^{\Phi}(X, Y)=N$. Then

$$
\left|\Phi^{\Phi}(f g)-\varphi^{\Phi}(f) \varphi^{\Phi}(g)\right| \leqq\left(\frac{2-\|K(\Phi)\|+N(1-\|K(\Phi)\|)}{(1-\|K(\Phi)\|)^{2}}\right)\|K(\Phi)\|^{N}\|f\|\|\| g \| .
$$

Proof. Using Lemma IV.1 and the series expansion

$$
\varphi^{\Phi}=\left(1-K(\Phi)^{*}\right)^{-1} \delta=\sum_{n=0}^{\infty} K(\Phi)^{* n} \delta
$$

we obtain

$$
\varphi^{\Phi}(f g)-\varphi^{\Phi}(f) \varphi^{\Phi}(g)=\sum_{n=N}^{\infty}\left(\delta\left(K(\Phi)^{n}(f g)\right)-\sum_{k=0}^{n} \delta\left(K(\Phi)^{k} f\right) \delta\left(K(\Phi)^{n-k} g\right)\right)
$$

so

$$
\begin{aligned}
& \left|\varphi^{\Phi}(f g)-\varphi^{\Phi}(f) \varphi^{\Phi}(g)\right| \leqq \sum_{n=N}^{\infty}(2+n)\|K(\Phi)\|^{n}\|f f\|\|\mid\| g\|\| \\
& =\left(\frac{2-\|K(\Phi)\|+N(1-\|K(\Phi)\|)}{(1-\|K(\Phi)\|)^{2}}\right)\|K(\Phi)\|^{N}\|f\|\|\| g\|\| .
\end{aligned}
$$


Acknowledgements. I would like to thank N. Kuiper and D. Ruelle for their hospitality at IHES, and G. Gallavotti, O. Lanford, D. Ruelle and especially W. Sullivan for many valuable discussions.

Remark. After the completion of this manuscript the author received a copy of [9], in which some of our results are obtained for the spin $-\frac{1}{2}$ model.

\section{References}

1. Dobrushin, R.L.: Theory of Probability and its Applications 13, 197-224 (1968)

2. Gallavotti, G., Miracle-Solé, S.: Commun. math. Phys. 7, 274-288 (1968)

3. Gruber, C., Merlini, D.: Physica 67, 308-322 (1973)

4. Hewitt,E., Ross, K.A.: Abstract Harmonic Analysis II. Berlin-Heidelberg-New York: Springer 1970

5. Krein, M.G.: Ukrain. Mat. Z. 1, $64-98$ (1949); 2, $10-59$ (1950). English translation: Amer. Math. Soc. Transl. Ser. 2, 34, 69-164 (1963)

6. Lanford,O.E., III: In: Statistical Mechanics and Mathematical Problems (1971 Battelle Rencontres). Lecture Notes in Physics, Vol. 20, A. Lenard, ed., pp. 1-113. Berlin-HeidelbergNew York: Springer 1973

7. Dunford, N.: Trans. Amer. Math. Soc. 44, 305-355 (1938)

8. Duneau, M., Iagolnitzer, D., Souillard, B.: J. Math. Phys. 16, 1662-1666 (1975)

9. Holley, J. A., Stroock, D. W.: Commun. math. Phys. 48, 249-265 (1976)

Communicated by G. Gallavotti

Received March 29, 1976 
